\title{
Dual adaptation of apparent concomitant motion contingent on head rotation frequency
}

\author{
ROBERT B. POST \\ University of California, Davis, California \\ and \\ ROBERT B. WELCH \\ NASA Ames Research Center, Mountain View, California
}

\begin{abstract}
Perceived movement of a stationary visual stimulus during head motion was measured before and after adaptation intervals during which participants performed voluntary head oscillations while viewing a moving spot. During these intervals, participants viewed the spot stimulus moving alternately in the same direction as the head was moving during either .25- or $2.0-\mathrm{Hz}$ oscillations, and then in the opposite direction as the head at the other of the two frequencies. Postadaptation measures indicated that the visual stimuli were perceived as stationary only if traveling in the same direction as that viewed during adaptation at the same frequency of head motion. Thus, opposite directions of spot motion were perceived as stationary following adaptation depending on head movement frequency. The results provide an example of the ability to establish dual (or "context-specific") adaptations to altered visual-vestibular feedback.
\end{abstract}

Under everyday viewing conditions, stationary visual stimuli appear to remain stationary during head motion, despite the changes in direction of the visual stimulus relative to the observer. This aspect of perception is variously termed "position constancy" (Hay \& Sawyer, 1969) or "constancy of visual direction" (Wallach \& Kravitz, 1965a, 1965b; see also Welch, 1978). However, if a small stimulus is viewed in an otherwise empty visual field, some deviation from position constancy may be perceived, an experience that has been termed "apparent concomitant motion" (ACM; Tietz \& Gogel, 1978).

It has been known since the work of Stratton $(1896,1897)$ that ACM is modifiable by exposure history. Specifically, if participants view stimuli that consistently move either in the same direction as head motion (with movement) or opposite to the head motion (against movement), the result is a tendency for subsequently viewed stationary objects to appear to move against or with the head, respectively (Hay, 1968, 1971; Hay \& Goldsmith, 1973; Post, 1997; Post \& Lott, 1992, 1993; Tietz \& Gogel, 1978; Wallach \& Floor, 1970; Wallach \& Frey, 1969; Wallach \& Kravitz, 1965a, 1965b). Accordingly, if participants are asked to select a target that appears to be stationary following such adaptation, they will tend to choose a stimulus that is moving in the same manner as that viewed during adaptation.

The vestibulo-ocular reflex (VOR) is also modifiable under similar conditions in which atypical image motion is paired with head motion. Exposure to with and against motion during head movement results in decreases and in-

We are grateful to Alison Glasser and Lori Siff for assistance in data collection. Correspondence should be addressed to R. B. Post, Department of Psychology, University of California, Davis, CA 95616 (e-mail: rbpost@ucdavis.edu). creases of VOR gain, respectively (Collewijn, Martins, \& Steinman, 1981; Gauthier \& Robinson, 1975; Gonshor \& Melvill Jones, 1971; Miles \& Eighmy, 1980; Post \& Lott, 1992). These gain changes are adaptive in that the VOR regains much of its ability to minimize image slip during the altered visual feedback. It is likely that the changes in ACM that occur when participants are exposed to stimulus motion contingent on head motion reflect the gain changes in the VOR (Hay, 1968, 1971; Post, 1997; Post \& Lott, 1993), although only a few investigators (e.g., Post \& Lott, 1992) have measured both types of adaptation in the same experiment.

Human participants reveal sensory and sensorimotor plasticity with respect to a great many other sensory rearrangements besides those involving ACM and the VOR (see Welch, 1978, for a review of this literature), the most common of which is lateral rotation of the visual field by wedge prisms (see, e.g., Held \& Hein, 1958). Furthermore, several studies, most of them recent (e.g., Baker, Perlmuter, Peterson, Rude, \& Robinson, 1987; Cunningham \& Welch, 1994; Flook \& McGonigle, 1977; Shelhamer, Robinson, \& Tan, 1992; Tan, Shelhamer, \& Zee, 1992; Welch, Bridgeman, Anand, \& Browman, 1993), have demonstrated that repeated alternation between adapting to a sensory rearrangement and "readapting" to the normal visual environment (or to a second sensory rearrangement) results in the acquisition of separate, simultaneously maintained adaptations. For example, Welch et al. (1993) found that repeated exposure and adaptation to 15 -diopter $\left(8.6^{\circ}\right)$ prismatic displacement to one side, alternating with exposure and adaptation to 15-diopter displacement in the opposite direction, eventually produced a more rapid adaptation to and recovery from each of these conditions than was the case the first time participants experienced them. The investigators referred to this phenomenon as "dual adaptation." Fur- 
thermore, when dual-adapted participants were then exposed to a "novel" (i.e., never previously experienced) prismatic displacement of 30 diopters $\left(17.1^{\circ}\right)$, they adapted more rapidly and completely than did a non-dual-adapted control group. This they termed "adaptive generalization."

The present paper describes a study aimed at establishing dual adaptation for ACM. Specifically, we examined whether opposite ACM adaptations could be established within individuals at different head oscillation frequencies. Post and Lott (1993) have demonstrated that ACM adaptation is frequency specific, as is true for VOR adaptation (Lisberger, Miles, \& Optican, 1983; Melvill Jones \& Gonshor, 1982). Additionally, Shelhamer et al. (1992) demonstrated that dual (or, as they referred to it, "context-specific") adaptation of the VOR can be established contingent on vertical gaze angle. The goal of the present experiment was to produce dual adaptation of ACM, contingent on headturning frequency. Thus, participants adapted to visual motion in opposite directions, each associated with a different frequency of head motion, and were tested at the same two frequencies to determine whether context-specific ACM adaptation was established.

\section{METHOD}

\section{Participants}

Eight adult volunteers, 20-42 years old, with normal oculomotor function, served as participants. Participants were either emmetropic or mildly myopic and could focus to the distance of the stimulus display.

\begin{abstract}
Apparatus
The experimental apparatus and stimuli have been described extensively previously (Post, 1997; Post \& Lott, 1992, 1993). Participants were seated facing a vertical hemicylindrical screen, with the head at the center of screen curvature (radius $=98 \mathrm{~cm}$ ). A spot of low-power laser light was projected onto the concave surface of the screen with a mirror galvanometer, which could produce horizontal motion of the spot that was coupled to participants' head motions. A potentiometer allowed either the experimenter or the participants to adjust both the amplitude (gain) and the sign of the phase of spot motion through a range from - .6 to .6 (spot motion/head motion).
\end{abstract}

\section{Procedure}

All participants engaged in two experimental sessions that were separated by at least 1 week. Each session consisted of three phases. In the first (preadaptation) phase, ACM measures were obtained. During the second (adaptation) phase, the participants viewed stimuli that alternately moved with and against their head motion. In the last (postadaptation) phase, ACM measures were obtained. A timeline depicting the course of experimental sessions is presented in Figure 1.

Preadaptation ACM measures. Three measures of ACM were recorded at the beginning of each experimental session during both $.25-$ and $2.0-\mathrm{Hz}$ head oscillations. Half the participants performed the $.25-\mathrm{Hz}$ measures first and the other half performed the $2.0-\mathrm{Hz}$ measures first. Prior to each measure, the room lights were extinguished and an electronic beeper was activated to pace participants' head movements. Participants voluntarily oscillated their heads left and right, as paced by the beeper, and fixated the laser spot on the hemicylindrical screen. Prior to each trial, the spot was adjusted by the experimenter to be moving slightly either in the same direction as head motion (with motion) or in the opposite direction from head motion (against motion). Participants adjusted the motion of the spot with the hand-held potentiometer until it appeared stationary during head motion. When participants perceived the spot to be stationary, the experimenter sampled $5 \mathrm{sec}$ of the motion of both the head and the spot. A potentiometer attached to the top of the participants' heads provided the head rotation signal and a copy of the analogue voltage driving the galvanometer provided the spot motion signal. These signals were digitized at $50 \mathrm{~Hz}$ and stored on disk for later analyses.

Adaptation. The adaptation phase lasted $25 \mathrm{~min}$, during which participants alternately oscillated their heads at $2.0 \mathrm{~Hz}$ for $30 \mathrm{sec}$ and at $.25 \mathrm{~Hz}$ for $2 \mathrm{~min}$ while attempting to fixate a moving spot stimulus. The different durations were used for the two frequencies of head movement to compensate partially for the substantially larger number of head movements that would occur at $2.0 \mathrm{~Hz}$ if equal durations were used. The spot moved in opposite directions during the two frequencies of head motion. In one session, the spot was moving with the head during the $2.0-\mathrm{Hz}$ motions and against the head during the $.25-\mathrm{Hz}$ motions. In the other session, the pairings of frequency and spot direction were reversed. The spot moved through $60 \%$ of the head motions during both the with and the against motions. Ten cycles of alternating with and against exposure occurred during the 25 min of adaptation.

Postadaptation ACM measures. Three measures were obtained at both head-movement frequencies in a manner identical to that used for the preadaptation measures. The frequency of head motion tested first was the same as during the preadaptation measures.

The recordings of head and spot motion that were obtained during the pre- and postadaptation ACM measures were analyzed by dividing each record of spot motion by the corresponding head motion record. The resulting value indicates by its sign whether the spot moved with or against the head, and by its magnitude the percentage of head motion through which it traveled (gain). The median of the three trials during both pre- and postadaptation measures for each movement frequency was saved for further analyses. This value corresponds to the physical motion that appears stationary and is therefore the stimulus required to nullify the illusory ACM.

\section{RESULTS}

The means of all participants' ACM nulling values are presented in Figure 2 for both experimental sessions. The top panel of the figure shows the results from the session in which the adaptation stimuli were $.25 \mathrm{~Hz}$ with motion alternating with $2.0 \mathrm{~Hz}$ against motion. The figure suggests that during the preadaptation measures, participants selected a slightly positive (with) nulling stimulus during $.25-\mathrm{Hz}$ oscillations and a slightly negative (against) stimulus during $2.0-\mathrm{Hz}$ oscillations. Following adaptation, the sign of the nulling stimuli remained the same while the gain increased. The bottom panel shows the results from the session in which the adaptation stimuli were $.25 \mathrm{~Hz}$ against motion alternating with $2.0 \mathrm{~Hz}$ with motion. As in the other session, participants' preadaptation measures were slightly positive (with) during .25- $\mathrm{Hz}$ oscillations and slightly negative (against) during $2.0-\mathrm{Hz}$ oscillations. The postadaptation measures, however, show a reversal of this tendency.

Separate 2 (measure: pre- or postadaptation) $\times 2$ (frequency: $.25 \mathrm{~Hz}$ or $2.0 \mathrm{~Hz}$ ) analyses of variance (ANOVAs) were conducted on the results from the two experimental sessions. For the session in which the adaptation stimuli were $.25 \mathrm{~Hz}$ with and $2.0 \mathrm{~Hz}$ against (Figure 2, top panel), the main effect of frequency was statistically significant $[F(1,7)=24.91, p<.01]$, as was the interaction of measure and frequency $[F(1,7)=12.36, p<.01]$. This analysis supports the trends evident in the figure. Participants 

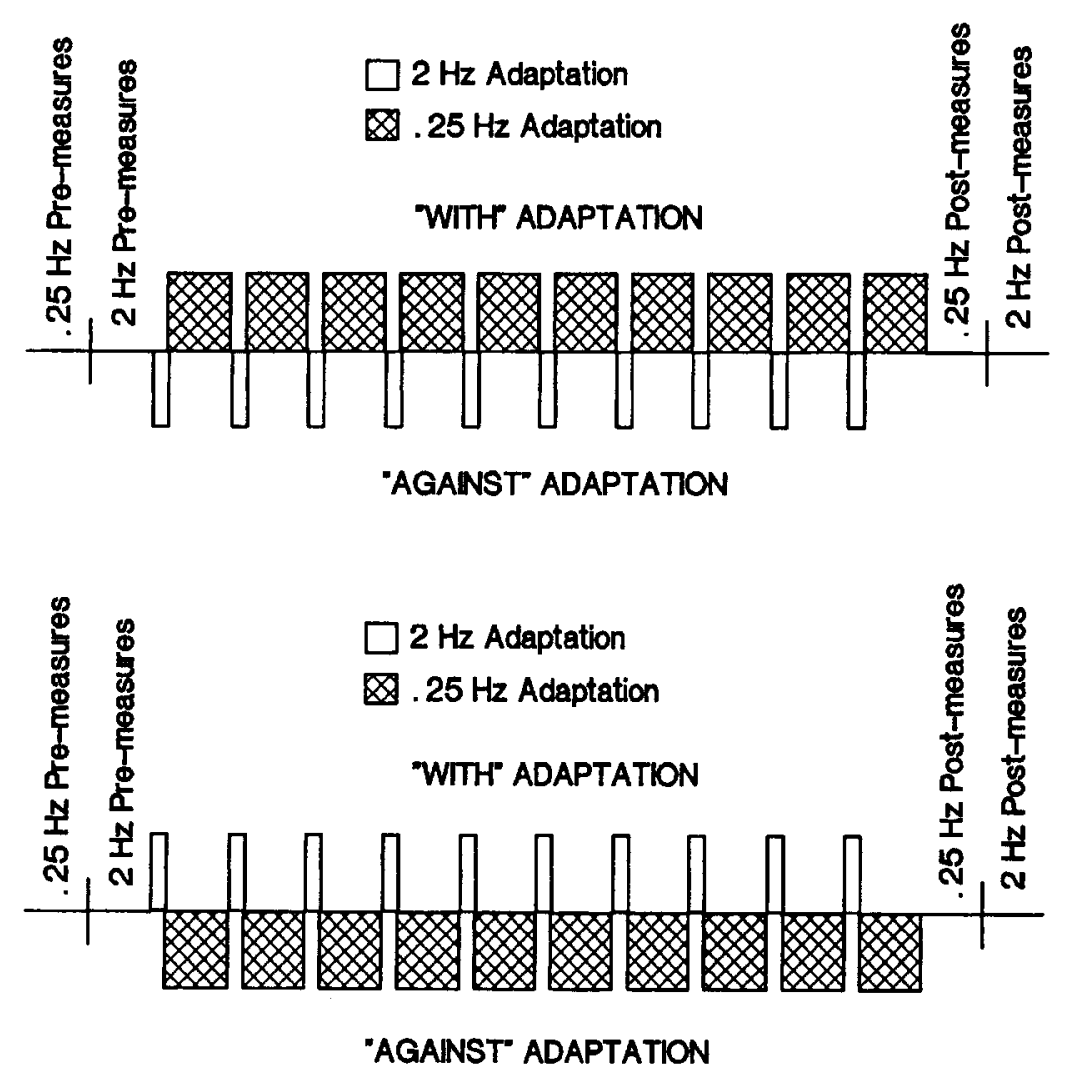

Figure 1. Timelines depicting the course of both experimental sessions. Top panel: Adaptation stimulus consisted of alternating against motion at $2.0 \mathrm{~Hz}$ for 30 sec and with motion at $.25 \mathrm{~Hz}$ for $2 \mathrm{~min}$. Bottom panel: The pairings of adaptation stimulus frequency and direction are reversed.

initially required a slight with nulling stimulus at $.25 \mathrm{~Hz}$ and an equally slight against nulling stimulus at $2.0 \mathrm{~Hz}$, whereas subsequent alternate viewing of with $.25-\mathrm{Hz}$ and against $2.0-\mathrm{Hz}$ stimuli strengthened this tendency. Planned comparisons revealed that the pre-post difference was statistically significant for the $.25-\mathrm{Hz}$ measures $(p<.05)$ and approached statistical significance for the $2.0-\mathrm{Hz}$ measures $(p=.052)$. For the session in which the adaptation stimuli were $.25 \mathrm{~Hz}$ against and $2.0 \mathrm{~Hz}$ with (Figure 2, bottom panel), neither main effect was statistically significant (both $p \mathrm{~s}>.05$ ). However, the interaction of measure and frequency was significant $[F(1,7)=12.20, p<01]$. This analysis thus supports the trends evident in the figure. It can be seen that participants initially required a slight with nulling stimulus at $.25 \mathrm{~Hz}$ and a slight against nulling stimulus at $2.0 \mathrm{~Hz}$, whereas subsequent alternate viewing of an against $.25-\mathrm{Hz}$ stimulus and a with $2.0-\mathrm{Hz}$ stimulus reversed this tendency. Planned comparisons revealed that the pre-post difference was statistically significant for both the .25 - and $2.0-\mathrm{Hz}$ measures (both $p$ s $<.05$ ). For the purposes of evaluating the participants' tendency in the preadaptation measures to select with and against nulling stimuli during $.25-\mathrm{Hz}$ and $2.0-\mathrm{Hz}$ movements, respectively, a separate 2 (session) $\times 2$ (frequency) ANOVA was conducted on the combined preadaptation measures from the two sessions. The main effect of frequency was statis- tically significant $[F(1,7)=9.70, p<.05]$, thus supporting the trends apparent in both panels of the figure.

\section{DISCUSSION}

The present results confirm that ACM is subject to dual adaptation. Thus, repeated pairing between a given head oscillation frequency $(.25$ or $2.0 \mathrm{~Hz})$ and a given form of rearranged visual feedback (with or against) led to the acquisition of a separate adaptation for each of these situations. The similarity of this observation for ACM and those obtained with the VOR (Baker, Perlmuter, et al., 1987; Baker, Wickland, \& Peterson, 1987; Shelhamer et al., 1992; Tan et al., 1992) strengthens the assumption that these two visual-vestibular processes are intimately (and perhaps causally) related.

An interesting secondary observation deserving comment is the finding that the preadaptation ACM nulling settings differed for the two head oscillation frequencies. This finding has been reported previously (Post \& Lott, 1993) and may be due to differences in VOR gain as a function of head-turning frequency. Specifically, VOR gain tends to be less than 1 between .1 and $.5 \mathrm{~Hz}$, and increases at higher frequencies of head rotation (Barr, Schultheis, \& Robinson, 1976; Benson, 1970; Keller, 1978). If the VOR gain is less than 1, the images of stationary objects tend to 


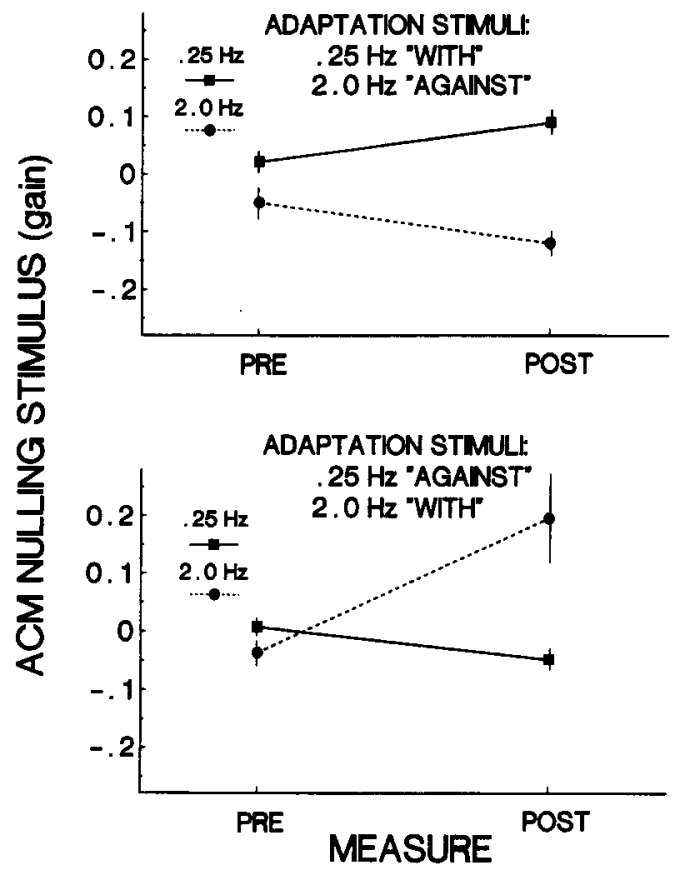

Figure 2. Apparent concomitant motion (ACM) nulling stimuli before and after adaptation during .25- and $2.0-\mathrm{Hz}$ head oscillations. Top panel: Adaptation stimulus consisted of alternating against motion at $2.0 \mathrm{~Hz}$ for $30 \mathrm{sec}$ and with motion at $.25 \mathrm{~Hz}$ for $2 \mathrm{~min}$. Bottom panel: The pairings of adaptation stimulus frequency and direction are reversed. Error bars represent $\pm 1 S E M$.

slip in the direction opposite head rotation and are therefore perceived as moving against, causing with nulling stimuli to be selected. At higher frequencies, if the VOR gain exceeds 1 , the opposite is true. An alternate interpretation of $\mathrm{ACM}$ posits that it results from errors in the perceived distance of the fixated stimulus due to the "specific distance tendency" (Gogel \& Tietz, 1973). An interpretation of the present findings within this framework would appear to require the specific distance tendency to change with the frequency of head motion. Since there is no reason to assume a priori that the specific distance tendency varies with frequency of head motion, an explanation of the present results in these terms appears untenable.

Although not examined in the present experiment, it would be interesting to determine whether the dual adaptation established by our procedure leads to adaptive generalization, as Welch et al. (1993) observed for prism adaptation. For example, this possibility could be examined by, first, eliminating any residual ACM adaptation to the most recent altered gain to which the dual-adapted participants have been confronted and then exposing them to a headfrequency-altered gain relationship that they have never experienced before. For example, in the present situation the adaptive generalization test might have entailed a $1-\mathrm{Hz}$ head-turning frequency coupled with a with spot motion that equaled $30 \%$ of the head motion. If the experience of adapting alternately to two opposite forms of altered gain somehow increases the dual-adapted participants' ability to adapt to new examples of altered gain, it should be seen as more rapid and/or complete $\mathrm{ACM}$ adaptation than is seen for a control group of non-dual-adapted participants.

Once dual adaptation has been demonstrated for a given visual or visual-motor process, two important questions arise: (1) What are the necessary conditions for this dual adaptation? and (2) What, exactly, has been changed? The first of these questions asks, in effect, how the brain "knows" when to invoke one type of adaptation and when to invoke another. Clearly, there must be available one or more stimuli that, as a result of the dual-adaptation training regimen, have acquired the status of discriminative cues for this "switching mechanism." An analysis of our situation reveals a number of possible cues, and since the present experiment was not designed to answer this question, any one or all of them might be responsible for our results. Further, these potential discriminative cues entail both external and self-generated stimuli. The former include (1) the sound of the electronic beeper (.25 vs. $2.0 \mathrm{~Hz})$ and (2) instructions to the participant that provided knowledge about the frequency with which they were to move the head.

Potential self-generated cues include (1) the differential efference required for the two kinds of head oscillations, (2) the differential vestibular signals generated by the oscillations, (3) the differential proprioceptive-kinesthetic feedback from these movements, and (4) the difference in the visual feedback (i.e., motion of the dot stimulus) contingent on the two head movement frequencies. A subtle difference between these two categories of discriminative cue is that the external cues (as well as efference) can serve as "warning signals" and, in principle, allow the adaptive mechanism to make its switch before the visual rearrangement is actually presented. On the other hand, if the necessary condition for changing to the alternate adaptation is the visual and/or proprioceptive-kinesthetic feedback (i.e., reafference) that occurs from interacting with the alternate visual environment, then, obviously, this shift cannot occur until the feedback has arrived at its appropriate neural locus (or loci). Under these circumstances, then, even very strongly dual-adapted participants will reveal a delay (perhaps very brief) between (1) the moment the visual environment has been changed and (2) the initiation of the appropriate adaptation. This situation is analogous to that of serial reversal learning (see, e.g., Harlow, 1949) in which, after repeated reversals of the response-reward contingency, participants learn to switch to the "new" contingency immediately after the second trial of this alternate contingency, the first trial being necessary to inform them that the rules have just been changed. Clearly, further research will be necessary to evaluate this field of possible sources of the dual adaptation obtained in the present experiment.

What is the end product of ACM dual adaptation? In the studies by Cunningham and Welch (1994) and Welch et al. (1993), both of which involved hand-eye correction in response to rearranged visual environments, one could entertain as one possibility that participants had acquired a conscious and deliberate strategy for changing their visual-motor behavior as soon as they were aware that the visual - motor contingency had been switched (or was about to be switched). Clearly, however, a voluntary strategy as 
an outcome of dual adaptation is not a useful concept for the present situation since it is very difficult to imagine how observers could deliberately change their experience of visual position constancy. Thus, the appropriate place to look for what has changed in the present situation is likely to be found at a lower level.

Given the strong similarities between ACM and VOR adaptation, it is interesting to note that different levels of explanation are typically invoked in the discussion of the mechanisms underlying these two forms of plasticity. Specifically, although most of the preceding discussion of ACM dual adaptation was in terms of conditioning theory, considerable work on VOR gain adaptation has been concerned with determining the physiological basis of the adaptive changes. This work has led to the isolation of particular neural structures and physiological mechanisms as strong candidates for the locus of modification (see, e.g., Lisberger, 1996; Peterson, Kinney, Quinn, \& Slater, 1996). The viewpoints and discussions are not mutually exclusive, however, but rather are concerned with different levels of explanation, and thus may serve as an example of how one instance of conditioning is achieved at a physiological level.

\section{REFERENCES}

Baker, J., Perlmuter, S. I., Peterson, B. W., Rude, F. R., \& RobinSON, F. R. (1987). Simultaneous opposing adaptive changes in cat vestibulo-ocular reflex direction for two body orientations. Experimental Brain Research, 69, 220-224.

Baker, J., Wickland, C., \& Peterson, B. (1987). Dependence of cat vestibulo-ocular reflex adaptation on animal orientation during adaptation and rotation in darkness. Brain Research, 408, 339-343.

Barr, C. C., Schultheis, W., \& Robinson, D. A. (1976). Voluntary non-visual control of the human vestibulo-ocular reflex. Acta Otolaryngologica, 81, 365-375.

Benson, A. J. (1970). Interactions between semicircular canals and graviceptors. In D. E. Busby (Ed.), Recent advances in aerospace medicine (pp. 249-261). Dordrecht: D. Reidel.

Collewijn, H., Martins, A. J., \& Steinman, R. M. (1981). The time course of adaptation of human compensatory eye movements. Documenta Ophthalmologica Proceedings Series, 30, 123-133.

Cunningham, H. A., \& Welch, R. B. (1994). Multiple concurrent visual-motormappings: Implications for models of adaptation. Journal of Experimental Psychology: Human Perception \& Performance, 20, 987-999.

Flook, J. P., \& MCGonigle, B. O. (1977). Serial adaptation to conflicting prismatic rearrangement effects in monkey and man. Perception, 6, 15-29.

Gauthier, G. M., \& Robinson, D. A. (1975). Adaptation of the human vestibulo-ocular reflex to magnifying lenses. Brain Research, 92, 331-335.

Gogel, W. C., \& Tietz, J. D. (1973). Absolute motion parallax and the specific distance tendency. Perception \& Psychophysics, 13, 284-292.

Gonshor, A., \& Melvill Jones, G. (1971). Plasticity in the adult human vestibulo-ocular reflex arc. Proceedings of the Canadian Federation of Biological Science, 14, 11.

Harlow, H. F. (1949). The formation of learning sets. Psychological Review, 56, 51-65.

HAY, J. C. (1968). Visual adaptation to an altered correlation between eye movement and head movement. Science, 160, 429-430.

HAY, J. C. (1971). Does head-movement feedback calibrate the perceived direction of optical motions? Perception \& Psychophysics, 10, 286-288.

HAY, J. C., \& GoldSMITH, W. M. (1973). Space-time adaptation of visual position constancy. Journal of Experimental Psychology, 99, 1-9.

HAY, J. C., \& SAWYER, S. (1969). Position constancy and binocular convergence. Perception \& Psychophysics, 5, 310-312.
Held, R., \& HEIN, A. (1958). Adaptation of disarranged hand-eye coordination contingent upon re-afferent stimulation. Perceptual \& Motor Skills, 8, 87-90.

KELLER, E. L. (1978). Gain of the vestibulo-ocular reflex in monkey at high rotational frequencies. Vision Research, 18, 311-315.

LISBERGER, S. G. (1996). Motor learning and memory in the vestibuloocular reflex: The dark side. In S. M. Highstein, B. Cohen, \& J. A. Buttner-Ennever (Eds.), New directions in vestibular research (Annals of the New York Academy of Sciences, Vol. 781, pp. 525-531). New York: New York Academy of Sciences.

Lisberger, S. G., Miles, F. A., \& Optican, L. M. (1983). Frequency selective adaptation: Evidence for channels in the vestibulo-ocular reflex? Journal of Neuroscience, 3, 1234-1244.

Melvill Jones, G., \& Gonshor, A. (1982). Oculomotor response to rapid head oscillation $(0.5-5.0 \mathrm{~Hz})$ after prolonged adaptation to vision reversal. Experimental Brain Research, 45, 45-58.

Miles, F. A., \& Eighmy, B. B. (1980). Long-term adaptive changes in primate vestibulo-ocular reflex: I. Behavioral observations. Journal of Neurophysiology, 43, 1406-1425.

Peterson, B. W., Kinney, G. A., Quinn, K. J., \& Slater, N. T. (1996). Potential mechanisms of plastic adaptive changes in the vestibuloocular reflex. In S. M. Highstein, B. Cohen, \& J. A. Buttner-Ennever (Eds.), New directions in vestibular research (Annals of the New York Academy of Sciences, Vol. 781, pp. 499-512). New York: New York Academy of Sciences.

Post, R. B. (1997). Adaptation to altered visual-vestibular feedback: Mechanisms of maintenance and recovery. Perception \& Psychophysics, 59, 148-154.

Post, R. B., \& LoTT, L. A. (1992). The relationship between vestibuloocular reflex plasticity and changes in apparent concomitant motion. Vision Research, 32, 89-96.

Post, R. B., \& Lotr, L. A. (1993). Frequency specificity in the adaptation of apparent concomitant motion. Bulletin of the Psychonomic Society, 31, 53-56.

Shelhamer, M., Robinson, D. A., \& Tan, H. S. (1992). Contextspecific adaptation of the gain of the vestibulo-ocular reflex in humans. Journal of Vestibular Research, 2, 89-96.

Stratton, G. (1896). Some preliminary experiments on vision without inversion of the retinal image. Psychological Review, 3, 611-617.

Stratton, G. (1897). Vision without inversion of the retinal image. Psychological Review, 4, 341-360, 463-481.

Tan, H. S., Shel hamer, M., \& ZeE, D. S. (1992). Effect of head orientation and position on vestibulo-ocular reflex adaptation. In B. Cohen, D. L. Tomko, \& F. Giredry (Eds.), Sensing and controlling motion: Vestibular and sensorimotor function (Annals of the New York Academy of Sciences, Vol. 656, pp. 158-165). New York: New York Academy of Sciences.

TIETZ, J. D., \& Gogel, W. C. (1978). Adaptation to apparent concomitant motion in the absence of physical or retinal motion. Bulletin of the Psychonomic Society, 12, 1-4.

WALLACH, H., \& FLOOR, L. (1970). On the relation of adaptation to field displacement during head movements to the constancy of visual direction. Perception \& Psychophysics, 8, 95-98.

WallaCh, H., \& FREY, K. J. (1969). Adaptation in the constancy of visual direction measured by a one-trial method. Perception \& Psychophysics, 5, 249-252.

Wallach, H., \& Kravitz, J. H. (1965a). The measurement of the constancy of visual direction and of its adaptation. Psychonomic Science, 2., 217-218

Wallach, H., \& Kravitz, J. H. (1965b). Rapid adaptation in the constancy of visual adaptation with active and passive rotation. Psychonomic Science, 3, 165-166.

WELCH, R. B. (1978). Perceptual modification: Adapting to altered sensory environments. New York: Academic Press.

Welch, R. B., Bridgeman, B., Anand, S., \& Browman, K. E. (1993). Alternating prism exposure causes dual adaptation and generalization to a novel displacement. Perception \& Psychophysics, 54, 195-204.

(Manuscript received May 19, 1997; revision accepted for publication June 19, 1997.) 